\title{
Death anxiety and its predictors among the companions of hospitalized patients with coronavirus disease 2019
}

\author{
Mehrooz Alishah $^{1}{ }^{\mathbb{D}}$, Masoumeh Bagheri-Nesami ${ }^{2,3}{ }^{\circledR}$, Seyed Robabeh Babaei ${ }^{1 * \mathbb{D}}$, Mehrnoosh Alishah $^{4}$ \\ 1Master of Special Nursing, Nasibeh School of Nursing and Midwifery, Mazandaran University of Medical Sciences, Sari, Iran \\ ${ }^{2}$ Professor, Traditional and Complementary Medicine Research Center, Addiction Institute, Mazandaran University of Medical \\ Sciences, Sari, Iran \\ ${ }^{3}$ World Federation of Acupuncture-Moxibustion Societies (WFAS), Beijing, China \\ ${ }^{4}$ Master of Information Science and Science of Mazandaran University of Medical Sciences, Sari, Iran
}

\begin{abstract}
Background and aims: Death anxiety (DA) is associated with stress and impaired immunity and thereby, puts individuals at risk for coronavirus disease 2019 (COVID-19). The aim of this study was to assess DA and its predictors among the companions of hospitalized patients with COVID-19.

Methods: This descriptive-analytical study was conducted in 2020 on 200 companions of patients with COVID-19 hospitalized in Imam Khomeini, BouAli, and Razi hospitals in Sari and Qaemshahr, Iran. Sampling was performed conveniently. Data were collected using a demographic and clinical characteristics questionnaire for patients, a demographic and clinical characteristics questionnaire for companions, and Templer DA Scale. The SPSS software (v. 21.0) was used for data analysis via the independent sample $t$ test, chi-square test, Pearson's correlation analysis, and multiple linear regression analysis.

Results: The mean score of DA was $47.21 \pm 10.62$ (in the possible range of 15-75). Participants' DA had significant relationship with their gender, their patients' anorexia and arterial oxygen saturation, and COVID-19 among other family members $(P<0.05)$. Participants' gender, patients' anorexia and arterial oxygen saturation, and COVID-19 among other family members significantly explained $0.066 \%$ of the total variance of DA $(P=0.002)$. The strongest predictors of DA were arterial oxygen saturation $(\beta=0.17 ; P=0.01)$ and gender $(\beta=0.15 ; P=0.0)$.

Conclusion: The companions of hospitalized patients with COVID-19 experience high levels of DA and hence, interventions are needed to reduce their DA.

Keywords: Death anxiety, Coronavirus disease 2019, Patient's companion
\end{abstract}

*Corresponding Author: Seyed Robabeh Babaei,

Email: robabe.babaei@gmail. com

Received: 17 December 2020 Accepted: 2 March 2021 ePublished: 30 June 2021

\section{Introduction}

Many different health problems have affected human health and life throughout history (1). Coronavirus disease 2019 (COVID-19) is one of these problems. COVID-19 was first diagnosed in December 31, 2020 in Wuhan, China, and rapidly turned into a pandemic (2). In its severe form, COVID-19 causes severe respiratory involvement (2) and symptoms such as fever, cough, shortness of breath, and dyspnea (3). The COVID-19 pandemic requires significant changes in life, causes numerous deaths, and necessitates social distancing (4). Statistics provided by the World Health Organization show that until July 2, 2021, the number of deaths due to COVID-19 was 3954324 and the number of afflicted people was 182319261 (5).

Thinking about death, either the death of self or others, is associated with an unpleasant feeling called death anxiety (DA) (6) which is the most basic type of anxiety among humans (7). DA is a reaction to chronic or lifethreatening diseases (8) and is caused due to the fact that there is no first-hand experience of death (9). The concept of DA is so important that the North American Nursing Diagnosis Association considers it as a main nursing diagnosis (10). DA is considered as a problem with increasing prevalence (11), particularly after the onset of the COVID-19 pandemic.

Caregiving to a beloved person with a serious disease can put heavy strain on family caregivers and cause them different problems (12) such as DA. High levels of DA can in turn negatively affect normal life (13). The results of a study showed that DA had significant negative relationship with quality of life among the caregivers of patients with cancer (14). Another study reported that most caregivers of patients with COVID-19 had anxiety (15). Similarly, two studies during the COVID-19 pandemic reported the high prevalence of anxiety and depression among the caregivers of patients with cerebral paralysis (16) and the caregivers of hospitalized children with COVID-19 (17).

The level of DA depends on personal characteristics and social factors such as age and gender $(18,19)$. For example,

(C) 2021 The Author(s); Published by Shahrekord University of Medical Sciences. This is an open-access article distributed under the terms of the Creative Commons Attribution License (http://creativecommons.org/licenses/by/4.0), which permits unrestricted use, distribution, and reproduction in any medium, provided the original work is properly cited. 
studies reported that elderly women had higher levels of DA than elderly men $(20,21)$. Nonetheless, despite the importance of DA and its significant effects on health and life, there is limited information about DA and its contributing factors during the COVID-19 pandemic. Therefore, the present study sought to assess DA and its predictors among the companions of hospitalized patients with COVID-19.

\section{Methods}

Design

This descriptive-analytical study was conducted in 2020 .

\section{Participants and setting}

Participants were 200 companions of patients with COVID-19 hospitalized in Imam Khomeini, BouAli, and Razi hospitals in Sari and Qaemshahr, Iran. Statistical population of the study consisted of all companions of patients with COVID-19 in the study setting. Sample size was determined using the results of a former study which reported that the prevalence of DA among critical care nurses was $25 \%$ (22). Accordingly, the sample size calculation formula (Eq. 1) showed that with a $d$ of 0.06 and a confidence interval of $0.95,200$ companions were needed for the study.

$n=\frac{\left(Z_{1-\alpha / 2}\right)^{2} P(1-P)}{d^{2}}=\frac{(1.96)^{2} 0.25(1-0.25)}{0.0036}=200$ Eq. (1)

Sampling was performed conveniently. Selection criteria were companionship with a hospitalized patient with COVID-19, first-degree kinship with the patient, agreement for participation, basic literacy skills, age over eighteen, no affliction by mental disorders or intake of anxiolytic agents, no affliction by serious health problems, and patient hospital stay of 2-14 days.

\section{Data collection}

Data collection tools were a demographic and clinical characteristics questionnaire for patients, a demographic and clinical characteristics questionnaire for companions, and Templer DA Scale. The items of the demographic and clinical characteristics questionnaire for patients were on gender, age, marital status, educational level, occupation, financial status, family size, COVID-19 symptoms, COVID-19 affliction among other family members, kinship with the companion, history of contact with a suspected case of COVID-19, type of underlying disease, number of hospitalization days, high-risk behaviors, insurance coverage, and vital signs. The items of the demographic and clinical characteristics questionnaire for companions were on age, gender, marital status, educational level, occupation, financial status, kinship with the patient, and place of residence. These two questionnaires were completed through the self-report method, interviewing with patients or their companions, and referring to patients' medical records.
Templer DA Scale was used for DA assessment. This scale was introduced in 1970 and contains fifteen items on death-related thoughts, fear over death, talking about death, and fear and thoughts about incurable diseases. Items are scored on a five-point scale from 1 ("Completely agree") to 5 ("Completely disagree"). Therefore, the total score of the scale is 15-75, with higher scores showing higher DA. The cutoff score of the scale is 45 (23-25). Scores less than 45 show low DA while scores more than 45 show high DA (26). A former study on 138 bachelor's students in Iran confirmed the acceptable validity and reliability of the Persian Templer DA Scale with content validity ratios of more than 0.76 , content validity indices of more than 0.68 , and a Cronbach's alpha of 0.83 (27). Cronbach's alpha in the present study was calculated using the data collected from forty companions and was 0.83 .

\section{Data analysis}

The SPSS software (v. 21.0) was used to analyze the data at a significance level of less than 0.05. Data were described using the measures of descriptive statistics, namely mean, standard deviation, absolute frequency, and relative frequency. Moreover, the independent-sample $t$ and the chi-square tests as well as the Pearson's correlation analysis were used for relationship analysis. The multiple linear regression analysis was also used to determine the predictors of DA.

\section{Results}

In total, 200 companions of patients with COVID-19 participated in the study. The mean of their age was $40.86 \pm 11.02$ in the range of $20-79$ years. Most of them were female $(61 \%)$, married $(82 \%)$, and children of patients (68.5\%), had moderate income (56.5\%), and lived in urban areas (65\%). Most patients were married (85\%), had moderate income (53\%), had insurance (93.5\%), and were head of household (60\%). Table 1 shows the characteristics of participants and their patients.

The most common symptoms of patients at the time of hospital admission were dyspnea (47.5\%), weakness (44.5\%), fever (34.5\%), cough (31\%), nausea (20.5\%),

Table 1. Participants' and their patients' demographic and clinical characteristics

\begin{tabular}{lccc}
\hline Characteristics & Mean \pm SD & Minimum & Maximum \\
\hline Companion's age $(y)$ & $40.86 \pm 11.02$ & 20 & 79 \\
Family size & $3.6 \pm 2.03$ & 1 & 10 \\
Length of hospital stay & $9.8 \pm 8.3$ & 2 & 45 \\
Arterial oxygen saturation & $93.4 \pm 4.5$ & 60 & 99 \\
Heart rate & $87.9 \pm 18.07$ & 114 & 182 \\
Body temperature & $37.08 \pm 0.56$ & 36 & 39 \\
Systolic blood pressure & $118.21 \pm 21.88$ & 75 & 240 \\
Diastolic blood pressure & $73.19 \pm 11.08$ & 40 & 100 \\
Respiratory rate & $19.39 \pm 3.70$ & 10 & 40 \\
Patient's age $(y)$ & $63.95 \pm 16.55$ & 16 & 96 \\
\hline
\end{tabular}


myalgia (20\%), and shivering (19\%). Other symptoms were vomiting (15\%), anorexia (14.5\%), altered consciousness (12\%), abdominal pain (11\%), headache (8.5\%), sore throat $(5.5 \%)$, increased level of serum creatinine $(4.5 \%)$, chest pain $(4.5 \%)$, dizziness $(4.5 \%)$, constipation $(2.5 \%)$, bloody diarrhea (1.5\%), hemoptysis (1\%), and palpitation $(0.5 \%)$. The most common symptoms developed during hospital stay were dyspnea (6\%) and increased level of serum creatinine $(3.5 \%)$. Moreover, the most common underlying diseases among patients were hypertension (46\%), diabetes mellitus (44\%), overweight and obesity (43\%), cardiovascular problems (39.5\%), hyperlipidemia (16\%), impaired immunity (16\%), renal failure (13.5\%), respiratory disease $(8.5 \%)$, and anemia $(7 \%)$, and $10 \%$ of patients had no underlying disease.

The mean score of DA was $47.21 \pm 10.62$ and the score of most participants was more than 45 (58.5\%). The mean score of DA had significant relationship with arterial oxygen saturation $(\mathrm{r}=0.167 ; P=0.018)$. Moreover, the mean score of DA in female participants was significantly greater than male participants $(48.61 \pm 10.55$ vs. $45.03 \pm 10.43 ; t=2.36 ; P=0.019)$. Affliction of other family members by COVID-19 also had significant relationship with participants' DA $\left(\chi^{2}=5.607 ; P=0.018\right)$. Other demographic characteristics of participants had no significant relationship with their DA $(P>0.05)$. In addition, patients' anorexia had significant relationship with participants' DA $\left(\chi^{2}=4.095 ; P=0.043\right)$, so that participants whose patients had anorexia reported lower DA (20\%). DA had no significant relationship with other clinical symptoms and underlying diseases of patients $(P>.05)$.

Multiple regression analysis using the Enter method revealed that participants' gender, patients' arterial oxygen saturation, patients' anorexia, and the affliction of other family members by COVID-19 significantly explained $0.066 \%$ of the total variance of DA $(P=0.002)$. The strongest predictors of DA were arterial oxygen saturation $(\beta=0.17 ; P=0.01)$ and gender $(\beta=0.15 ; P=0.0)$ (Table 2$)$.

\section{Discussion}

The aim of this study was to assess DA and its predictors among the companions of hospitalized patients with COVID-19. Findings showed that more than half of the participants had high DA and the level of DA among female participants was significantly more than male participants. Similarly, a previous study reported that female family caregivers of patients with cancer had higher levels of DA (14). It seems that there is no real difference between men and women regarding DA and the observed differences are due to the fact that cultural factors make women more openly express their anxiety (28).

Study findings also showed that DA had significant relationship with arterial oxygen saturation. Arterial oxygen saturation is an indicator of COVID-19 severity and its low level indicates more severe COVID-19 (29) and more serious COVID-19 complications such as acute respiratory distress syndrome, septic shock, and death (30). This interesting finding may be due to the fact that patients with lower arterial oxygen saturation may have altered consciousness and be hospitalized in critical care units, where their companions have limited access, if any, to data on arterial oxygen saturation. Contrarily, as patients with higher arterial oxygen saturation are in general hospital wards, their companions have easier access to data on arterial oxygen saturation and hence, may experience more DA in response to fluctuations in arterial oxygen saturation.

We also found a significant relationship between DA among participants and COVID-19 affliction among other family members, so that participants whose other family members had COVID-19 had lower DA. Similarly, a previous study reported that the caregivers of patients with hemodialysis who gave care to several patients had lower anxiety than those who gave care just to one patient (31). Lower DA among caregivers with several COVID-19 afflicted family members may be due to their greater knowledge and experience about COVID-19.

Study findings indicated anorexia as the only symptom with significant relationship with DA, so that participants whose patients had anorexia reported lower or no DA. This finding may be due to the fact that most patients who did not have anorexia experienced gastrointestinal problems such as nausea and vomiting after eating which resulted in anxiety for their companions. On the other hand, patients with anorexia received intravenous fluid therapy and did not experience gastrointestinal problems and hence, their companions experienced lower anxiety.

Our findings also showed no significant relationship between participants' age and their DA. A former study also reported the same finding (32), while two studies on the caregivers of patients with cancer and healthy adult people found that younger people had higher DA $(14,33)$. The insignificant relationship of age with DA in the present study may be due to the significant effects of the COVID-19 pandemic on all age groups and the high

Table 2. The results of the regression analysis to determine the predictors of DA

\begin{tabular}{|c|c|c|c|c|c|}
\hline Variables & Beta & Standard error & Standardized beta & $t$ & $P$ value \\
\hline Constant & 9.295 & 15.197 & & 0.612 & 0.541 \\
\hline Companion's gender & -3.453 & 1.491 & -0.159 & -2.316 & 0.022 \\
\hline Arterial oxygen saturation & 0.418 & 0.162 & 0.177 & 2.580 & 0.011 \\
\hline Affliction of other family members by COVID-19 & 3.448 & 1.822 & 0.130 & 1.893 & 0.060 \\
\hline Anorexia & -3.310 & 2.065 & -0.110 & -1.603 & 0.111 \\
\hline
\end{tabular}


risk of affliction by COVID-19 among people from all age groups.

Study findings also revealed that $90 \%$ of patients with COVID-19 had underlying diseases, mainly cardiovascular disease, diabetes mellitus, and obesity. The results of a meta-analysis also showed hypertension, cardiovascular disease, and diabetes mellitus as the most prevalent underlying diseases among patients with COVID-19 (34). Studies in China, Iran, and Italy reported affliction by underlying diseases as a major risk factor for COVID-19 and a significant predictor of COVID-19 severity (29,35-36). These findings highlight that patients with underlying diseases may have impaired immunity and hence, should take effective measures to prevent affliction by COVID-19.

Another finding of the present study was the insignificant relationship of DA with educational level. A previous study also found the same finding (37), while another study revealed that educational level and occupational position had significant relationship with DA (38). People with higher educational level usually obtain more information about COVID-19, its mortality rate, and its affliction risk from media and their colleagues and hence, may experience higher levels of anxiety. Further studies are needed to produce more reliable results regarding the relationship of educational level and DA in the COVID-19 pandemic.

Some participants experienced great grief at the time of responding to the data collection tools and were reluctant to respond to the tools. This might have been due to their high level of DA. Similarly, a study reported that nurses with high level of anxiety were reluctant to talk with their patients and family members about death (39).

\section{Conclusion}

This study shows high levels of DA among the companions of hospitalized patients with COVID-19, particularly among female companions, those with COVID-19 affliction among other family members, and those whose patients have higher levels of arterial oxygen saturation. Therefore, educational and supportive interventions are needed to improve knowledge about COVID-19, its complications, and its treatments among patients' companions. Online educational methods for patient and family education may be safer and produce better results in the current COVID-19 pandemic.

\section{Study limitations}

This study did not assess all factors with potential effects on companions' DA, such as patients' COVID-19 severity and hospitalization in critical care units. Futures studies are recommended to evaluate the effects of these factors on DA among the companions of patients with COVID-19. Moreover, interventional studies are needed to evaluate the effects of different interventions on DA.

\section{Acknowledgement}

We would like to thank the Research Administration of Mazandaran
What does this paper contribute to the wider global clinical community?

The companions of patients with COVID-19 experience high levels of DA and some of them equate COVID-19 affliction with death. Therefore, effective communication between these companions and healthcare providers and providing clear answers to their questions may reduce their DA. Nurses and nursing students should be trained about quality patient and family education and should be encouraged to use these educations in their daily practice in order to reduce companions' anxiety and DA. Effective informational support for family members and companions of patients with COVID-19 is also necessary to improve their knowledge and reduce their DA. Information technology, telephone-based followup programs, online caregiving guidelines, group support, and psychological counseling can be used to reduce stress and anxiety among family members and companions.

University of Medical Sciences, Sari, Iran, for supporting this study. Moreover, we are thankful to the nurses of Imam Khomeini, BouAli, and Razi hospitals in Sari and Qaemshahr, Iran.

\section{Conflict of Interests}

The authors declare no conflict of interests.

\section{Ethical Approval}

The Ethics Committee of Mazandaran University of Medical Sciences, Sari, Iran, approved this study (code: IR.MAZUMS. REC.1399.7402). Participants were informed about the study aim, confidentiality of data management, voluntariness of participation, and freedom to unilaterally withdraw from the study. Informed consent was obtained from all participants.

\section{References}

1. Bao Y, Sun Y, Meng S, Shi J, Lu L. 2019-nCoV epidemic: address mental health care to empower society. Lancet. 2020;395(10224):e37-e8. doi: 10.1016/s01406736(20)30309-3.

2. National guidelines for new coronavirus. Ministry of Health and Medical Education; February 2019. [Persian].

3. Huang C, Wang Y, Li X, Ren L, Zhao J, Hu Y, et al. Clinical features of patients infected with 2019 novel coronavirus in Wuhan, China. Lancet. 2020;395(10223):497-506. doi: 10.1016/s0140-6736(20)30183-5.

4. Evans R, Wilner PJ, Spillane K. Caring for our caregivers in body, mind and spirit during the COVID-19 pandemic. Patient Exp J. 2020;7(2):129-35.

5. WHO Coronavirus disease 2019 (COVID-19) Situation Report-68. https://www.who.int/emergencies/diseases/novelcoronavirus-20192020(pengf.tulyang).

6. Hlubocky FJ, Muffly LS, Gomez JX, Breitenbach K, Cella D, McNeer JL, et al. Death anxiety, psychological distress, and quality of life (QOL) in adolescent and young adult (AYA) cancer patients with hematologic malignancies in early survivorship. J Clin Oncol. 2016;34(15 Suppl):10073. doi: 10.1200/JCO.2016.34.15_suppl.10073.

7. Fritsche I, Jonas E, Kayser DN, Koranyi N. Existential threat and compliance with pro-environmental norms. J Environ Psychol. 
2010;30(1):67-79. doi: 10.1016/j.jenvp.2009.08.007.

8. Lok GKI, Ng MWI, Zhu MMX, Chao SKK, Li SX. Mediating effect of religious belief on death anxiety in Chinese adolescents: a cross-sectional study. Int J Sch Health. 2019;6(4):14-20. doi: 10.30476/intjsh.2019.45884.

9. Salehi F, Mohsenzade F, Arefi M, Salehi Zahabi S, Amirifard $\mathrm{N}$. Death anxiety in patients with cancer in Kermanshah. Iran J Cancer Care. 2020;1(1):24-9. doi: 10.29252/ijca.1.1.24. [Persian].

10. Carpenito-Moyet LJ. Nursing Care Plans \& Documentation: Nursing Diagnoses and Collaborative Problems. 5th ed. Lippincott Williams \& Wilkins; 2009.

11. Mahbobi M, Etemadi M, Khorasani E, Ghiasi M. The relationship between spiritual health and social anxiety in chemical veterans. J Mil Med. 2012;14(3):186-91. [Persian].

12. Heyland DK, Dodek P, Rocker G, Groll D, Gafni A, Pichora $\mathrm{D}$, et al. What matters most in end-of-life care: perceptions of seriously ill patients and their family members. CMAJ. 2006;174(5):627-33. doi: 10.1503/cmaj.050626.

13. Gire J. How death imitates life: cultural influences on conceptions of death and dying. Online Readings in Psychology and Culture. 2014;6(2):3.

14. Soleimani MA, Lehto RH, Negarandeh R, Bahrami N, Chan $\mathrm{YH}$. Death anxiety and quality of life in Iranian caregivers of patients with cancer. Cancer Nurs. 2017;40(1):E1-E10. doi: 10.1097/ncc.0000000000000355.

15. Rahimi T, Dastyar N, Rafati F. Family caregivers' experiences of caring for patients with COVID-19: a phenomenological study. 2020;3(1):95-109. doi: 10.21203/rs.3.rs-107195/v1.

16. Farajzadeh A, Dehghanizadeh $M$, Maroufizadeh S, Amini $M$, Shamili A. Predictors of mental health among parents of children with cerebral palsy during the COVID-19 pandemic in Iran: a web-based cross-sectional study. Res Dev Disabil. 2021;112:103890. doi: 10.1016/j.ridd.2021.103890.

17. Yuan R, Xu QH, Xia CC, Lou CY, Xie Z, Ge QM, et al. Psychological status of parents of hospitalized children during the COVID-19 epidemic in China. Psychiatry Res. 2020;288:112953. doi: 10.1016/j.psychres.2020.112953.

18. Shiekhy S, Issazadegan A, Basharpour S, Maroei Millan F. The relationship between death obsession and death anxiety, with hope among the nursing students of Urmia medical sciences university. J Urmia Nurs Midwifery Fac. 2013;11(6):410-8. [Persian].

19. Buzzanga VL, Miller HR, Perne SE, Sander JA, Davis SF. The relationship between death anxiety and level of self-esteem: a reassessment. Bull Psychon Soc. 1989;27(6):570-2. doi: 10.3758/bf03334672.

20. Ghufran M, Ansari S. Impact of widowhood on religiosity and death anxiety among senior citizens. J Indian Acad Appl Psychol. 2008;34(1):175-80.

21. Depaola SJ, Griffin M, Young JR, Neimeyer RA. Death anxiety and attitudes toward the elderly among older adults: the role of gender and ethnicity. Death Stud. 2003;27(4):335-54. doi: 10.1080/07481180302904.

22. Dadgari F, Rouhi M, Farci Z. Evaluation of death anxiety in nurses of intensive care units of selected AJA hospitals. Military Care Sciences. 2015;2(3):150-7. [Persian]

23. Seyedoshohadaee $M$, Bozorgi Matin $M$, Haghani $H$. Association of death anxiety and quality of life in the caregivers of patients with multiple sclerosis. Iran Journal of Nursing. 2019;32(120):1-13. doi: 10.29252/ijn.32.120.1. [Persian].
24. Peters L, Cant R, Payne S, O'Connor M, McDermott F, Hood K, et al. How death anxiety impacts nurses' caring for patients at the end of life: a review of literature. Open Nurs J. 2013;7:1421. doi: 10.2174/1874434601307010014.

25. Soleimani MA, Tabiban S, Bakhshande H, Asghary M. Effect of illness perception intervention on death anxiety in hemodialysis patients: a randomized clinical trial. J Mazandaran Univ Med Sci. 2018;28(162):12-24. [Persian].

26. Templer DI. The construction and validation of a death anxiety scale. J Gen Psychol. 1970;82(2d Half):165-77. doi: 10.1080/00221309.1970.9920634.

27. Rajabi GR, Bohrani M. Item factor analysis of the death anxiety scale. J Psychol. 2002;5(4):331-44. [Persian].

28. Fink G. Encyclopedia of Stress. 5th ed. Academic Press; 2000.

29. Guan WJ, Ni ZY, Hu Y, Liang WH, Ou CQ, He JX, et al. Clinical characteristics of coronavirus disease 2019 in China. N Engl J Med. 2020;382(18):1708-20. doi: 10.1056/NEJMoa2002032.

30. Ai JW, Zhang HC, Xu T, Wu J, Zhu M, Yu YQ, et al. Optimizing diagnostic strategy for novel coronavirus pneumonia, a multi-center study in Eastern China. medRxiv. 2020. doi: 10.1101/2020.02.13.20022673.

31. Lashgari F, Azimian J, Ranjbaran M, Soleimani MA. Predictive factors of death anxiety in caregivers of hemodialysis patients. Avicenna J Nurs Midwifery Care. 2019;27(3):187-97. doi: 10.30699/ajnmc.27.3.187. [Persian].

32. Masoudzadeh A, Setareh J, Mohammadpour RA, Modanloo Kordi M. A survey of death anxiety among personnel of a hospital in Sari. J Mazandaran Univ Med Sci. 2008;18(67):8490. [Persian].

33. Krause N, Pargament KI, Ironson G. In the shadow of death: religious hope as a moderator of the effects of age on death anxiety. J Gerontol B Psychol Sci Soc Sci. 2018;73(4):696703. doi: 10.1093/geronb/gbw039.

34. Emami A, Javanmardi F, Pirbonyeh N, Akbari A. Prevalence of underlying diseases in hospitalized patients with COVID-19: a systematic review and meta-analysis. Arch Acad Emerg Med. 2020;8(1):e35

35. Gholampour Y, Tehranineshat B, Najafi H, Farjam M, Rahimi Z, Bijani M. A study of demographic characteristics, clinical manifestations, radiologic and lab findings of patients hospitalized with COVID-19 in the south of Iran. J Adv Biomed Sci. 2020;10(3):2456-65. [Persian].

36. Riccardo F, Ajelli M, Andrianou XD, Bella A, Del Manso $M$, Fabiani $M$, et al. Epidemiological characteristics of COVID-19 cases in Italy and estimates of the reproductive numbers one month into the epidemic. medRxiv. 2020. doi: 10.1101/2020.04.08.20056861.

37. Bahrami N, Moradi M, Soleimani M, Kalantari Z, Hosseini F. Death anxiety and its relationship with quality of life in women with cancer. Iran Journal of Nursing. 2013;26(82):5161. [Persian].

38. Hasan Zehi E. Prediction of Death Anxiety in Elderly Based on Demographic Characteristics and Spiritual Components [thesis]. School of Educational Sciences and Psychology, Shiraz University; 2012. [Persian].

39. Deffner JM, Bell SK. Nurses' death anxiety, comfort level during communication with patients and families regarding death, and exposure to communication education: a quantitative study. J Nurses Staff Dev. 2005;21(1):19-23. doi: 10.1097/00124645-200501000-00005 